Article

\title{
An Efficient Heuristic Algorithm for Solving Connected Vertex Cover Problem in Graph Theory
}

\author{
Yongfei Zhang ${ }^{1}$, Jun Wu ${ }^{1}$, Liming Zhang ${ }^{2,3}$, Peng Zhao ${ }^{1}$, Junping Zhou ${ }^{1,2, *}$ and Minghao Yin ${ }^{1,2}$ \\ 1 College of Information Science and Technology, Northeast Normal University, Changchun, 130117, China \\ 2 Key Laboratory of Symbolic Computation and Knowledge Engineering of Ministry of Education, \\ Changchun 130012, China \\ 3 College of Computer Science and Technology, Jilin University Changchun 130012, China \\ * Correspondence: zhoujp877@nenu.edu.cn
}

Version January 25, 2018 submitted to

\begin{abstract}
The connected vertex cover $(C V C)$ problem is a variant of the vertex cover problem, which has many important applications, such as wireless network design, routing and wavelength assignment problem, etc. A good algorithm for the problem can help us improve engineering efficiency, cost savings and resources in industrial applications. In this work, we present an efficient algorithm GRASP-CVC (Greedy Randomized Adaptive Search Procedure for Connected Vertex Cover) for $C V C$ in general graphs. The algorithm has two main phases, i.e., construction phase and local search phase. To construct a high quality feasible initial solution, we design a greedy function and a restricted candidate list in the construction phase. The configuration checking strategy is adopted to decrease the cycling problem in the local search phase. The experimental results demonstrate that GRASP-CVC is competitive with the other competitive algorithm, which validate the effectivity and efficiency of our GRASP-CVC solver.
\end{abstract}

Keywords: Heuristic algorithm; connected vertex cover; GRASP.

\section{Introduction}

The connected vertex cover(CVC) is one of the classical combinatorial optimization problems, which was first introduced by Garey and Johnson in paper [1]. The problem not only shows its great importance in theory, but also has many significant industrial applications [2-5]. For example, in the wireless newtwork design, the vertices of the newtwork are connected by transmission links. We want to place a minimum number of relay stations on vertices such that any pair of relay stations are connected and every transmission link is incident to a relay station. This is the most direct application of the connected vertex cover model in the industry. Designing a good algorithm to solve this problem can not only improve work efficiency, save money and labor cost, but also save natural resources and reduce material waste. The problem is known to be NP-hard even in the planar 2-connected graph of maximum degree 4 [6], planar bipartite graph with maximum degree 4 [7], as well as in 3-connected graph [8].

The CVC problem has been studied for a long time, and a lot of efforts have been devoted to it. To date, there are mainly two types of algorithms to solve $C V C$, i.e., exact algorithms and approximation algorithms. All existing exact algorithms for CVC are mainly FPT (fixed-parameter tractable) algorithms in theory and these theoretical results are obtained in worst case. For example, Moser [2] showed that CVC is fixed-parameter tractable using the tree width as a parameter and proposed a dynamic programming algorithm running in $O\left(2^{w} \cdot w^{3 w+2} \cdot n\right)$ time, where $w$ is the tree width, $n$ is the number of nodes of nice tree decomposition. With the desired vertex cover size $k$ as parameter, Richter et al. [9] proposed an improved algorithm with running time in $\mathrm{O}\left(2.7606^{k}\right)$ in the worst case. Binkele-Raible [10] provided a better exact algorithm with running time in $O\left(2.4882^{k}\right)$ in the worst case. Because these exact algorithms fail to solve large graphs, a lot of efforts on approximation algorithms have been devoted. In the general graph, Savage [11] proposed the first constant ratio 
algorithm and proved that the set of internal nodes of any depth-first search tree is a solution of 2-approximation for $C V C$ problem. In addition, Fujito and Doi [12] proposed a 2-approximation algorithm for solving CVC, which runs in $O\left(\log ^{2} n\right)$ time using $O\left(\delta^{2}(m+n) / \log n\right)$ processors on an EREW-PRAM, where $n$ is the number of vertices, $m$ the number of edges, and $\delta$ is the maximum vertex degree. Fernau and Manlove [7] proved that CVC is NP-hard to approximate within $10 \sqrt{5}-21$ in general graphs unless $P=N P$. Therefore, it is difficult to improve the approximation ratio of approximation algorithms in general graphs, which makes the researchers change their research angle into the special graphs. Escoffier et al. [13] proved that the CVC problem is APX-complete in bipartite graphs of maximum degree 4 and is polynomial time solvable in chordal graphs. In addition, they also showed that CVC is 5/3-approximable for a class of special graphs (where solving the minimum vertex cover problem used polynomial time) and a polynomial time approximation algorithm for CVC in planar graphs was presented. Cardinal and Levy [14] proposed an approximation algorithm in dense graphs and the algorithm approximated the CVC problem with a ratio strictly less than 2 in dense graphs. The first polynomial time approximation algorithm in unit disk graphs for $C V C$ problem was proposed in [3]. Li et al.[15] proved that the CVC problem is still NP-hard for 4-regular graphs and provided a lower bound for the problem. Moreover, they proposed two approximation schemes for this problem in 4-regular graphs with approximation ratio $3 / 2$ and $4 / 3+O(1 / n)$, respectively. Although the exact algorithms for $C V C$ can provide an optimal solution, they are hard and time consuming to deal with large scale instances. Furthermore, although some approximation algorithms for CVC can get good performance in special graphs, they are usually not suitable for dealing with general graphs, and the state-of-the-art approximation methods in general graphs can only provide an approximate ratio 2, which is often not enough in practice. This yields a new challenge for us to devise a heuristic algorithm for $C V C$ that can deal with large general graphs and obtain the best possible approximate solutions within a reasonable time.

In this article, the heuristic algorithm GRASP-CVC for CVC in general graphs is proposed and this algorithm can obtain a relatively good solution within a reasonable time. The heuristic algorithm GRASP-CVC is based on the framework of greedy randomized adaptive search procedure (GRASP) [16]. The algorithm GRASP-CVC has two main phases, i.e., construction phase and local search phase. The GRASP-CVC tries to construct a feasible initial solution greedily in the construction phase. During this phase, we design a greedy function to help to evaluate the benefit of adding a vertex to the current solution. Besides, we construct a restricted candidate list $(R C L)$ to assist in constructing a high quality initial solution in the construction phase. Then the initial solution is further improved in the local search phase. To prevent the local search from suffering severe cycling problem, the configuration checking (CC) strategy is adopted in the search. Relying on the CC, we avoid many unnecessary searches during the local search procedure and the efficiency of the GRASP-CVC is improved greatly. Once the local search phase cannot explore a better solution anymore, which means the local search phase reaches a local optima, the GRASP-CVC then restarts a new iteration and repeats the construction and local search phases until reaching the maximum iteration times. The best found solution will be the final solution after all iterations used up. The results of experiments demonstrate that GRASP-CVC provides better solutions compared to the competitive algorithm, which validate the effectivity and efficiency of our GRASP-CVC solver. Moreover, the GRASP-CVC obtains almost the same size solutions in 10 times running, which demonstrates GRASP-CVC is stable.

The rest of this paper is structured as follows. Some relevant definitions and background knowledge will be introduced in next section. In Section 3, the algorithm GRASP-CVC will be introduced and the two main components will be discussed in details. Experimental evaluations and analyses will be shown in Section 4. Conclusions and future work will be given in the last section.

\section{Preliminaries}

In this section, some definitions and background knowledge are provided. From now on, unless otherwise stated, we only consider the CVC problem on an undirected graph $G=(V, E)$, where 


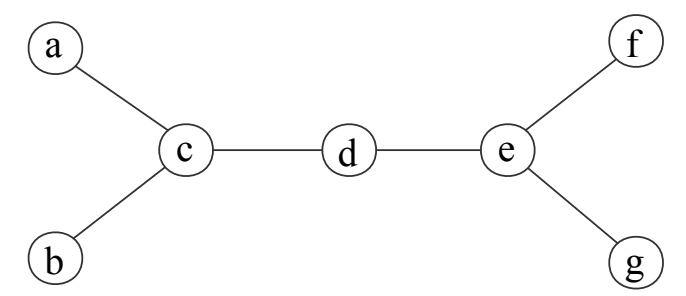

(a)

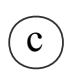

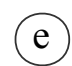

(b)

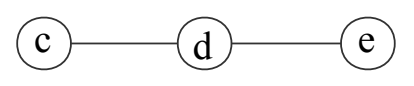

(c)

Figure 1. An example for MVC and CVC.

$V=\left\{v_{1}, v_{2}, \ldots, v_{n}\right\}$ is the vertices set and $E=\left\{e_{1}, e_{2}, \ldots, e_{m}\right\}$ is the edges set. In addition, each edge $e_{i}=\left(v_{k}, v_{j}\right)(1 \leqslant i \leqslant m, 1 \leqslant k, j \leqslant n, k \neq j)$ is a 2-element tuple on $V$ and we define vertices $v_{k}$ and $v_{j}$ are the endpoints of edge $e_{i}$. For a vertex subset $C \subseteq V$ and an edge $e_{i}$, if $C$ contains no endpoint of $e_{i}$, we say $e_{i}$ is uncovered by $C$, otherwise, we say $e_{i}$ is covered by $C$.

Definition 1. (Vertex Cover, $V C)$ Given a graph $G=(V, E)$, a subset of vertices $C \subseteq V$ is a vertex cover $(V C)$ of $G$ if each edge in $E$ has at least one endpoint in $C$.

Definition 2. (Minimum Vertex Cover, $M V C$ ) Given a graph $G=(V, E)$, the minimum vertex cover $(M V C)$ problem is to compute a VC of minimum cardinality in $G$.

Definition 3. (Induced Sub-graph, IS) Given two graph $G=(V, E)$ and $G^{\prime}=\left(V^{\prime}, E^{\prime}\right)$ where $V^{\prime} \subseteq V$ and $E^{\prime}=\left\{(v, u) \mid v, u \in V^{\prime} \wedge(v, u) \in E\right\}, G^{\prime}$ is called an induced sub-graph (IS) of $G$.

Definition 4. (Connected Graph) A graph is a connected graph if there is a path between every pair of vertices.

Definition 5. (Connected Vertex Cover, CVC) Given a connected graph $G=(V, E)$, the connected vertex cover (CVC) problem is to determine a subset $C \subseteq V$ with minimum cardinality such that $C$ satisfies the following two conditions: 1) $C$ is a vertex cover; 2) the IS induced by $C$ is a connected graph.

In order to facilitate the readers to understand the concepts given above, we provide an example in Figure 1. Figure 1(a) is a graph $G=(V, E)$, where $V=\{a, b, c, d, e, f, g\}$ and $E=\{(a, c),(b, c),(c, d),(d, e),(e, f),(e, g)\}$. Figure 1(b) presents an induced sub-graph by the vertex set $V_{1}^{\prime}=\{c, e\}$, which is just the solution of the $M V C$ problem of $G$. Because the induced sub-graph in Figure $1(\mathrm{~b})$ is not connected, the vertex set $V_{1}^{\prime}=\{c, e\}$ is not the solution of the CVC problem of $G$. By adding a vertex $d$ to $V_{1}^{\prime}$, we obtain a new vertex set $V_{2}^{\prime}=\{c, d, e\}$. The sub-graph induced by $V_{2}^{\prime}$ is shown in Figure 1(c). From Figure 1(c), we can notice that the vertex set $V_{2}^{\prime}$ is the minimal vertex subset that satisfies: 1 ) sub-graph induced by $V_{2}^{\prime}$ is connected; 2) the vertex set $V_{2}^{\prime}$ covers all edges in $E$. Thus, $V_{2}^{\prime}$ is a solution for the $C V C$ problem of $G$. From the example, we see that the size of the optimal $M V C$ solution provides a lower bound for the size of the optimal $C V C$ solution. In the following, we will present the conclusion in Theorem 1.

Theorem 1. The size of the optimal MVC solution provides a lower bound for the size of the optimal CVC solution. 
Proof. Given an undirected graph $G$, we suppose the size of the optimal $M V C$ solution is $N$ and the size of the optimal $C V C$ solution is $M$. Then we will analyze the theorem cases individually.

Case 1. There is a connected optimal solution of $M V C$. Under this circumstance, the $M V C$ solution is also a $C V C$ solution. Thus, we have $M=N$.

Case 2. There is no connected solution among all of the optimal solutions of $M V C$. Under this condition, it is impossible that $M=N$. Then we will prove the conclusion by using reduction to absurdity. Suppose there exists a $C V C$ solution that $M \leqslant N$. Under this condition, according to the definitions of $C V C$ and $M V C$, we know the $C V C$ solution is an $M V C$ solution as well. So, we get one $M V C$ solution whose size is less than $N$. However, this is contradiction with the previous assumption that $N$ is the size of the optimal $M V C$ solution.

In total, we finally reach the conclusion that $M \geqslant N$, which means that the optimal $M V C$ solution size provides a lower bound for the size of the optimal $C V C$ solution.

\section{GRASP-CVC algorithm for CVC}

GRASP-CVC (Greedy Randomized Adaptive Search Procedures for Connected Vertex Cover problem) is a multi-start meta-heuristic, which consists of two main phases: construction phase and local search phase. An initial solution is constructed firstly in the construction phase, and then the local search phase attempts to find the existence of a better solution by exploring the neighborhood of the initial solution. The two phases are executed repeatedly until reaching the termination condition, and then the GRASP-CVC takes the best found solution as the final output. The pseudo code of GRASP-CVC is outlined in Algorithm 1. At first, the solution $C^{*}$ is initialized (line 1). Then the algorithm enters an iteration loop (line 2-8). In each iteration, an initial CVC solution $C$ is generated firstly by the construction procedure (line 3), and then in the local search procedure (line 4), GRASP-CVC starts its search from $C$ trying to find a better solution. If $C$ possesses fewer vertices than the current best solution $C^{*}, C^{*}$ will be updated by $C$ (line 5-6). When the GRASP-CVC reaches the maximum iteration times, $C^{*}$ will be returned as the final solution (line 9). During the construction, we design a greedy function and construct a restricted candidate list to help to construct a high quality feasible solution. Moreover, in the local search, we adopt the configuration checking to reduce the cycling problem. The two phases will be discussed in details in the next two subsections.

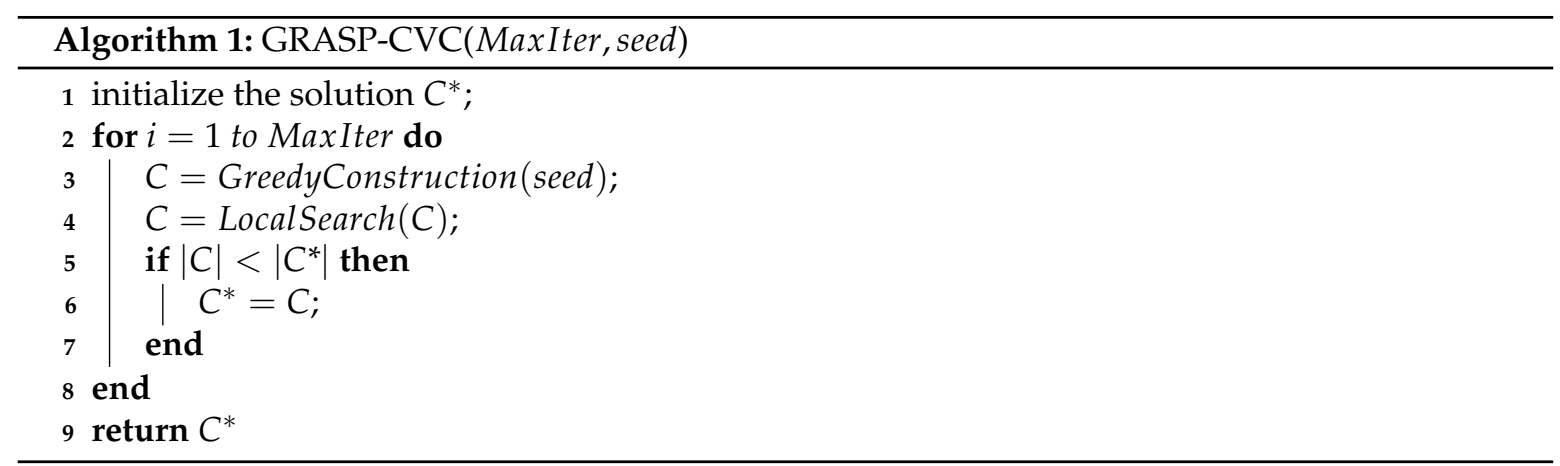

\subsection{Construction phase}

Before introducing the construction phase, we shall give the definitions of greedy function and restricted candidate list $(R C L)$ that play important roles in the construction phase. 
145

\subsubsection{Greedy function and RCL}

To evaluate the benefit of adding $v$ to the current solution, a greedy function score $(v)$ is designed, which is quite important for the construction of $R C L$ as well. In order to introduce the greedy function, we firstly propose some relevant definitions. We say vertices $u$ and $v$ are neighbors each other if there is an edge between them, and we use $N(u)=\{v \mid(u, v) \in E\}$ denoting the neighbors set of vertex $u$. The neighbor vertices set of a solution $C$, denoted as $N(C)$, can be calculated as follows.

$$
N(C)=\{v \mid v \notin C, v \in N(u), u \in C, N(u)=\{v \mid(u, v) \in E\}\}
$$

For a given vertex $v$, the greedy function $\operatorname{score}(v)$ can be calculated by the Formula (2).

$$
\operatorname{score}(v)=\operatorname{cost}(C)-\cos t\left(C^{\prime}\right)
$$

In the Formula (2), $C$ is the current solution. If $v$ in $C, C^{\prime}=C \backslash\{v\}\left({ }^{\prime} \backslash\right.$ means removing the vertex $v$ from $C$ ), otherwise, $C^{\prime}=C \cup\{v\}$. Moreover, $\cos t(C)$ is also a function calculated by the Formula (3).

$$
\operatorname{cost}(C)=\mid\{e \mid e \text { is not covered by } C\} \mid
$$

From the formula, we can know that the function $\operatorname{cost}(C)$ is to compute the total number of edges uncovered by $C$. In addition, when $v$ in $C$, $\operatorname{score}(v)$ is a negative number.

Using the greedy function $\operatorname{score}(v)$, we can identify those vertices that are most beneficial to the current solution. Moreover, the greedy function is an indispensable part in the construction of $R C L$.

The RCL consists of the vertices that are most beneficial to the current solution $C$. In the construction phase, one vertex is chosen randomly from $R C L$. And to construct a feasible initial solution, we select vertices from $R C L$. The construction of $R C L$ is described in Formula (4)

$$
R C L=\{v \mid v \in N(C) \wedge \operatorname{score}(v) \geqslant \operatorname{score}(u), \forall u \in N(C)\}
$$

Clearly, the elements of $R C L$ are the vertices set that having the highest score in the premise of not destroying the connectivity of $C$.

\subsubsection{Construction procedure}

After the necessary descriptions of greedy function and $R C L$, we shall discuss the greedy construction procedure in details. The greedy construction procedure GreedyConstruction is outlined in Algorithm 2.

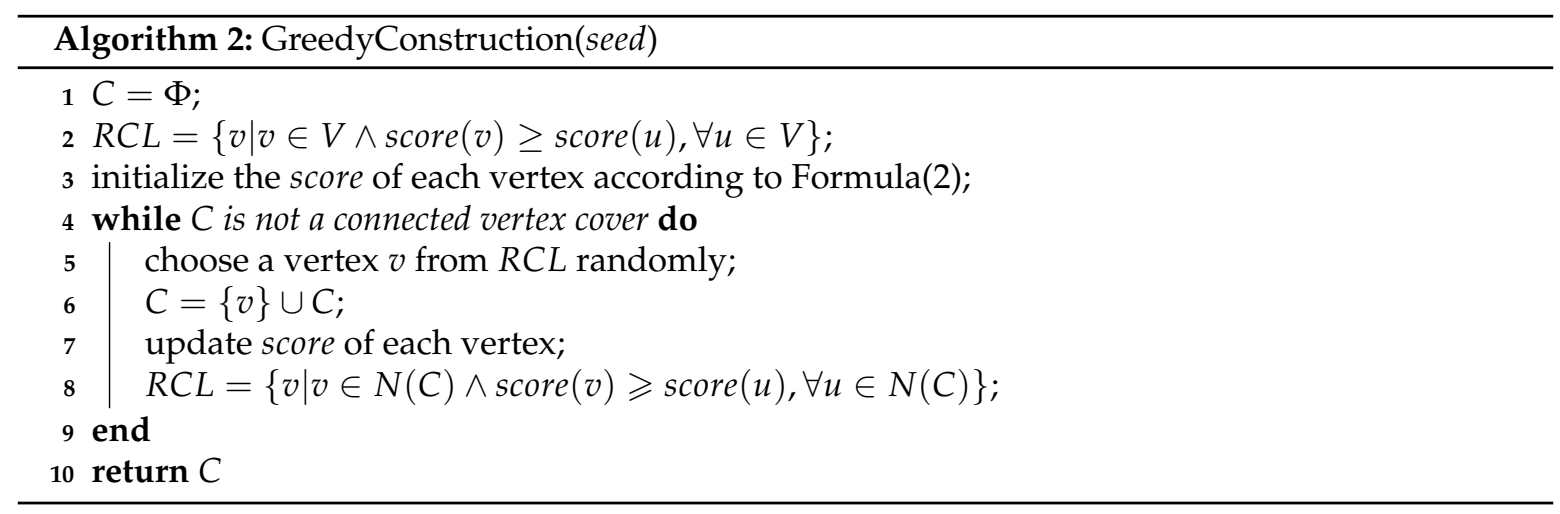

In the beginning, the connected vertex cover $C$ is initialized (line 1 ) and the score of each vertex is initialized according to the Formula (2) (line 2). The $R C L$ is initialized to the vertices with the highest score among the vertices set $V$ (line 3). Then, the procedure enters the main loop (line 4-8). In each 
loop, a vertex $v$ is chosen from the $R C L$ randomly and added into the construction solution $C$ (line 5-6). After the operations of line 5 and 6 , the score of each vertex is updated according to the Formula (2) (line 7). At the end of the loop, the $R C L$ is updated (line 8). The loop is executed until $C$ is constructed to be a solution of $C V C$, and then $C$ will be returned at the end of the construction procedure (line 10).

\subsection{Local search phase}

In this subsection, we shall introduce the configuration checking $(C C)$ strategy and discuss the working process of the local search phase in details.

\subsubsection{Configuration checking strategy}

Greedy strategy is usually an important part in the local search algorithms. It helps to lift the performance of the local search algorithms on large and hard instances. However, the greedy strategy usually also makes the local search algorithms easier to fall into the cycling problem (which means the algorithm visits the same part of the solution space repeatedly). Up to now, many efficient strategies have been used to handle this problem [17-20]. Configuration checking (CC) [21] strategy is one of those strategies and has been applied to some problems successfully [21-25]. Therefore, we adopt the CC strategy to avoid the cycling problem in the local search.

Before introducing the $C C$, we shall give the concept of vertex state. The vertex state of a vertex $v$ refers to the fact whether $v$ is located in the current solution. We can use a Boolean value 1 to represent $v$ is in the current solution and 0 to represent is not in. The configuration of a vertex $v$ is the states of all its neighbor vertices, which can be denoted by a n-dimensional Boolean vector $c_{v}$ (where $n$ is the number of neighbor vertices of $v$ ).

The main idea of the CC strategy is that a vertex $v$ is forbidden to add back to the current solution if its configuration keeps unchanged since it was removed from the current solution last time. This strategy is intuitive and reasonable in avoiding cycling problem, as it prevents the search facing the same scenario again. For example, for a $v \in C$, suppose its configuration is $c_{v 0}=(0,1,0,1)$ after removing $v$ out of $C$, then after several steps of searching, $v$ is selected again according to the greedy function value and suppose its configuration is $c_{v 1}$ now. If $c_{v 0}=c_{v 1}$, i.e. configuration not changed, if add $v$ back to the $C$, then the search goes back to the same situation before last time removing $v$ out of $C$ and the same solution space will be searched repeatedly. However, this situation will not occur when $c_{v 0} \neq c_{v 1}$, i.e. configuration changed(e.g. $\left.c_{v 1}=(1,1,0,1)\right)$. A Boolean array Change is used to implement the CC strategy. The element in the array Change is Change[v], which denotes whether the configuration of vertex $v$ is changed since it was removed from the current solution last time. We use Change $[v]=1$ expressing change, and Change $[v]=0$ expressing no change. Only the vertex $v$ whose Change $[v]=1$ is allowed to be added to the current solution in the local search process. In the process of local search, the values of Change are updated according the rules below [21].

- Rule 1: In the beginning, for each vertex $v$, set Change[v] to 1 .

- Rule 2: When removing $v$ from $C$, reset Change[v] to 0 .

- Rule 3: When $u$ changes its state, for each $v \in \mathrm{N}(u) \backslash \mathrm{C}$, Change[v] is set to 1 .

\subsubsection{Local search procedure}

In this subsection, the local search procedure is discussed at length. The main steps of the LocalSearch are listed in Algorithm 3.

The local search procedure performs as follows. In the first place, all elements of the Change are initialized to 1 (line 1), which means that all vertices are allowed to be added to the current solution in the beginning. Next, the local optimal solution $C^{*}$ is initialized as $C$, where $C$ is generated by the construction phase (line 2). Then, in the loop (line 3-17), the LocalSearch $(C)$ checks the feasibility of the current solution $C$. If $C$ is a $C V C$ solution which has a smaller vertex number than $C^{*}$, then $C^{*}$ will be updated by $C$ and a vertex $v$ with the highest score will be removed from $C$, and then the procedure 


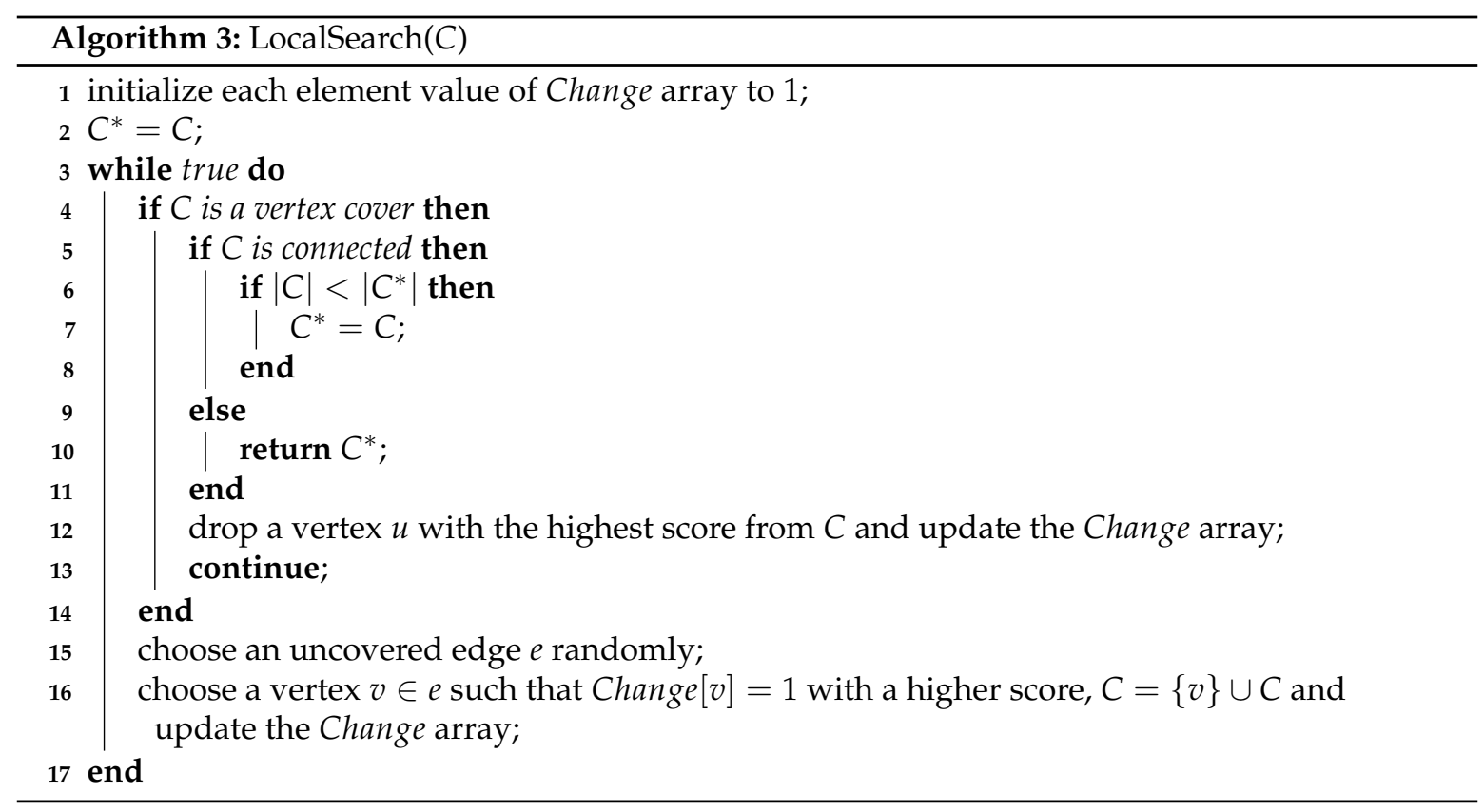

starts the next cycle (line 4-14). If $C$ is a $V C$ but not a $C V C$ anymore after $v$ is removed, which means the procedure reaches a local optimal solution, then $C^{*}$ will be returned as the final result of the local search procedure (line 9-11). If $C$ is not a $V C$ anymore after $v$ is removed, the procedure chooses an uncovered edge $e$ randomly, then chooses a vertex $v \in e$ such that Change $[v]=1$ with a higher score, and adds to $C=\{v\} \cup C$ (line 15-16). The Change array is updated according to Rule 2 and Rule 3 when a vertex changes its state (line 12,16).

\subsection{Time complexity analysis}

In this subsection, we discuss the time complexity of the main components of the GRASP-CVC algorithm.

First, we consider the algorithm for constructing the initial solution. In each loop, we need to update the score for $|V|$ vertices, and scan $|N(C)|$ vertices in order to update the $R C L$. If every time we add a vertex to $C$, an average of $d$ edges are covered. Then, we can get a solution in $O(|E| / d *(|V|+|N(C)|))=O(|E| *|V|)$.

Next, we consider the time complexity of the local search algorithm. In this part, there are three operations that affect the running time of the algorithm: drop a vertex from $C$ (Algorithm 3 , line 12), choose an uncovered edge (Algorithm 3, line 15), and update the Change array (Algorithm 3, line 12 and 16). Since the number of vertex in $C$ is $|C|$, the first operation can be done in a time of $O(|C|)$. In our implementation, we maintain a set to record uncovered edges, so an uncovered edge can be chosen in $O(1)$. The time complexity of the third operation depends on the degree of the operated vertex, and therefore this work can be done in $\mathrm{O}(\delta)$, where $\delta$ is the maximum degree of the vertices. Thus, the time complexity of the local search is $O(|C|+1+\delta)=O(|C|+\delta)$.

Overall, the run-time complexity of the GRASP-CVC is $O(|E| *|V|+|C|+\delta)$.

\section{Computational experiments}

During this section, the effectivity and efficiency of the GRASP-CVC algorithm are evaluated by performing some comparison experiments. We compare the GRASP-CVC algorithm with the currently best approximate algorithm (2-approximation algorithm) we know in general graphs [12]. Owing to the researches on CVC problem mainly focused on theoretical studies, there is no available 
approximation $C V C$ solver, so we implement the 2-approximation algorithm proposed in [12]. We implement the algorithms GRASP-CVC, and the 2-approximation algorithm in the C++ programming language. All of the experiments are carried on a work station under windows 7 operating system, 3.30GHZ CPU and 8GB memory.

\subsection{Benchmark instances}

In the experiments, we choose two well-known benchmarks in the field of $M V C$ research, the DIMACS and BHOSLIB instance sets. DIMACS benchmark contains both structured and randomized instances. The structured instances are generated from practical problems, such as coding theory, Keller conjecture and so on. The randomized instances are generated from the stochastic models, such as brock instances. The scale of these problem instances is from 50 vertices and 1000 edges to more than 5500 vertices and 5 million edges. BHOSLIB benchmark is famous for its hardness. The benchmark instances are transformed from $S A T$ instances that are generated in the phase transition area. And the instances in the phase transition area have been proved hard. From the two benchmarks, we select 37 DIMACS benchmark instances and 40 BHOSLIB benchmark instances, which are all employed in the best $M V C$ solver [25].

\subsection{Experiment parameter settings}

Before reporting the experimental results, we shall introduce some parameter settings.

In GRASP-CVC, there are two main parameters: maximum number of iterations (MaxIter) and random seed (seed). According to our experimental experience, we set Maxiter to 5000 and random seed to an interval from 1 to 10 . In order to evaluate the robustness of GRASP-CVC, we execute ten times for each instance using different random seeds.

For the sake of comparison between GRASP-CVC and 2-approximation algorithm for CVC, we execute ten times independent runs for the approximation algorithm as well. In addition, since the 2-approximation algorithm runs very fast, there is no need to set the cut-off time for the 2-approximation algorithm, we just set the cut-off time for GRASP-CVC to 1000 seconds.

\subsection{Experimental results}

In this subsection, we will provide the computational results of GRASP-CVC (GRASP-CVC) and the 2-approximation algorithm (2-Aprox) on the two chosen benchmarks. In the results, we provide the following information: the number of vertices and edges of each instance $(|V|,|E|)$, the best known size of the $M V C$ solution $(M V C)$, the best solution size solved by the corresponding algorithms (best), the average size of solutions solved by the corresponding algorithms $(a v g)$, the average time consumed by the corresponding algorithms (time). In addition, the MVC size with star $\left({ }^{*}\right)$ has been proved to be the optimal size of $M V C$ solution. 
Table 1. Experimental result on DIMACS Instances

\begin{tabular}{|c|c|c|c|c|c|c|c|c|}
\hline \multirow{2}{*}{ Instance } & \multirow{2}{*}{$|V|,|E|$} & \multirow{2}{*}{ MVC } & \multicolumn{3}{|c|}{ 2-Aprox } & \multicolumn{3}{|c|}{ GRASP-CVC } \\
\hline & & & time & best & avg & time & best & avg \\
\hline brock200_2.mis & 200,10024 & $188^{*}$ & 0.0003 & 198 & 199.7 & 0.0388 & 190 & 190 \\
\hline brock200_4.mis & 200,6811 & $183^{*}$ & 0.0003 & 195 & 197.2 & 1.3976 & 184 & 184 \\
\hline brock400_2.mis & 400,20014 & $371^{*}$ & 0.0007 & 396 & 397.5 & 6.1956 & 376 & 376 \\
\hline brock400_4.mis & 400,20035 & $367^{*}$ & 0.0006 & 396 & 396.6 & 10.2863 & 376 & 376 \\
\hline brock800_2.mis & 800,111434 & $776^{*}$ & 0.0017 & 798 & 798.2 & 35.0407 & 780 & 780 \\
\hline brock800_4.mis & 800,111957 & $774^{*}$ & 0.0017 & 798 & 798.1 & 173.9981 & 780 & 780 \\
\hline C125.9.mis & 125,787 & $91^{*}$ & 0.0002 & 116 & 117.4 & 0.003 & 91 & 91 \\
\hline C250.9.mis & 250,3141 & $206^{*}$ & 0.0005 & 240 & 243.2 & 2.3818 & 207 & 207 \\
\hline C500.9.mis & 500,12418 & $443^{*}$ & 0.0006 & 491 & 494.2 & 12.051 & 448 & 448 \\
\hline C1000.9.mis & 1000,49421 & 932 & 0.0012 & 994 & 995.7 & 66.33 & 939 & 939 \\
\hline C2000.9.mis & 2000,199468 & 1920 & 0.004 & 1992 & 1992.3 & 96.854 & 1933 & 1933 \\
\hline C2000.5.mis & 2000,999164 & 1984 & 0.0085 & 1998 & 1998 & 942.5859 & 1986 & 1986.1 \\
\hline C4000.5.mis & 4000,3997732 & 3982 & 0.0313 & 3998 & 3998.2 & 400.23 & 3986 & 3986 \\
\hline DSJC500.5.mis & 500,62126 & $487^{*}$ & 0.001 & 498 & 499.6 & 2.419 & 487 & 487 \\
\hline DSJC1000.5.mis & 1000,249674 & $985^{*}$ & 0.0025 & 998 & 999.8 & 887.5232 & 986 & 986 \\
\hline gen200_p0.9_44.mis & 200,1990 & $156^{*}$ & 0.0004 & 189 & 192.8 & 0.0583 & 164 & 164 \\
\hline gen200_p0.9_55.mis & 200,1990 & $145^{*}$ & 0.0003 & 186 & 191.5 & 0.0607 & 156 & 156 \\
\hline gen400_p0.9_55.mis & 400,7980 & $345^{*}$ & 0.0007 & 388 & 391.5 & 72.4805 & 358 & 358 \\
\hline gen400_p0.9_65.mis & 400,7980 & $335^{*}$ & 0.0006 & 389 & 390.9 & 18.8135 & 354 & 354 \\
\hline gen400_p0.9_75.mis & 400,7980 & $325^{*}$ & 0.0009 & 391 & 393.1 & 2.8003 & 357 & 357 \\
\hline hamming8 - 4.mis & 256,11776 & $240^{*}$ & 0.0005 & 255 & 255.9 & 0.0154 & 240 & 240 \\
\hline hamming $10-4 . m i s$ & 1024,89600 & $984^{*}$ & 0.0017 & 1023 & 1023.5 & 273.0134 & 990 & 990 \\
\hline keller4.mis & 171,5100 & $160^{*}$ & 0.0003 & 170 & 170.1 & 3.9266 & 160 & 160 \\
\hline keller5.mis & 776,74710 & $749^{*}$ & 0.0015 & 775 & 775.6 & 4.6351 & 756 & 756 \\
\hline keller6.mis & 3361,1026582 & 3302 & 0.0107 & 3360 & 3360.1 & 82.1559 & 3324 & 3324 \\
\hline MANN_a27.mis & 378,702 & $252^{*}$ & 0.0007 & 290 & 293.8 & 0.0475 & 260 & 260 \\
\hline MANN_a45.mis & 1035,1980 & $690^{*}$ & 0.0011 & 765 & 766.4 & 0.8462 & 704 & 704 \\
\hline MANN_a81.mis & 3321,6480 & 2221 & 0.0034 & 2353 & 2357.6 & 5.1007 & 2241 & 2241 \\
\hline p_hat $300-1 . m i s$ & 300,33917 & $292^{*}$ & 0.0006 & 298 & 298 & 0.1078 & 292 & 292 \\
\hline p_hat300 - 2.mis & 300,22922 & $275^{*}$ & 0.0004 & 298 & 298 & 0.642 & 275 & 275 \\
\hline p_hat $300-3$. mis & 300,11460 & $264^{*}$ & 0.0003 & 294 & 296 & 0.338 & 264 & 264 \\
\hline p_hat700-1.mis & 700,183651 & $689^{*}$ & 0.0019 & 698 & 699.8 & 7.7827 & 689 & 689 \\
\hline p_hat700-2.mis & 700,122922 & $656^{*}$ & 0.0017 & 700 & 700 & 1.792 & 656 & 656 \\
\hline p_hat700-3.mis & 700,61640 & $638^{*}$ & 0.0013 & 694 & 695.9 & 176.3111 & 638 & 638 \\
\hline p_hat1500 - 1.mis & 1500,839327 & $1488^{*}$ & 0.0074 & 1500 & 1500 & 19.7254 & 1489 & 1489.1 \\
\hline p_hat $1500-2 . m i s$ & 1500,555290 & $1435^{*}$ & 0.0051 & 1498 & 1498.1 & 27.6126 & 1438 & 1438 \\
\hline p_hat $1500-3$. mis & 1500,277006 & 1406 & 0.0033 & 1496 & 1496 & 210.4793 & 1409 & 1409 \\
\hline
\end{tabular}


Table 2. Experimental result on BHOSLIB Instances

\begin{tabular}{|c|c|c|c|c|c|c|c|c|}
\hline \multirow{2}{*}{ Instance } & \multirow{2}{*}{$|V|,|E|$} & \multirow{2}{*}{ MVC } & \multicolumn{3}{|c|}{ 2-Aprox } & \multicolumn{3}{|c|}{ GRASP-CVC } \\
\hline & & & time & best & avg & time & best & avg \\
\hline frb30 - $15-1$. mis & 450,17827 & $420^{*}$ & 0.001 & 449 & 449.6 & 11.3735 & 424 & 424 \\
\hline frb30-15-2.mis & 450,17874 & $420^{*}$ & 0.0006 & 447 & 448.4 & 7.873 & 425 & 425 \\
\hline frb30-15-3.mis & 450,17809 & $420^{*}$ & 0.0007 & 449 & 449.7 & 13.4266 & 424 & 424 \\
\hline frb30-15-4.mis & 450,17831 & $420^{*}$ & 0.0006 & 448 & 449.3 & 15.2355 & 424 & 424 \\
\hline frb30 - $15-5$. mis & 450,17794 & $420^{*}$ & 0.0005 & 448 & 449.3 & 25.6118 & 423 & 423 \\
\hline frb35-17-1.mis & 595,27856 & $560^{*}$ & 0.0011 & 592 & 593.7 & 29.4486 & 565 & 565 \\
\hline frb35-17-2.mis & 595,27847 & $560^{*}$ & 0.0009 & 592 & 592.8 & 8.5406 & 565 & 565 \\
\hline frb35-17-3.mis & 595,27931 & $560^{*}$ & 0.001 & 592 & 592.7 & 2.7005 & 565 & 565 \\
\hline frb35-17-4.mis & 595,27842 & $560^{*}$ & 0.001 & 592 & 593.6 & 172.9833 & 565 & 565 \\
\hline frb35-17-5.mis & 595,28143 & $560^{*}$ & 0.0009 & 594 & 594.5 & 34.4835 & 565 & 565 \\
\hline frb40-19-1.mis & 760,41314 & $720^{*}$ & 0.0012 & 758 & 759 & 187.7404 & 728 & 728 \\
\hline frb $40-19-2 . m i s$ & 760,41263 & $720^{*}$ & 0.0012 & 757 & 758 & 213.6616 & 726 & 726 \\
\hline frb $40-19-3 . m i s$ & 760,41095 & $720^{*}$ & 0.0012 & 758 & 759.2 & 101.0795 & 725 & 725 \\
\hline frb $40-19-4 . m i s$ & 760,41605 & $720^{*}$ & 0.0012 & 757 & 758.1 & 8.3877 & 726 & 726 \\
\hline frb $40-19-5 . m i s$ & 760,41619 & $720^{*}$ & 0.0014 & 758 & 759 & 33.3235 & 725 & 725 \\
\hline frb $45-21-1 . m i s$ & 945,59186 & $900^{*}$ & 0.0015 & 944 & 944.4 & 665.5343 & 908 & 908 \\
\hline frb $45-21-2 . m i s$ & 945,58624 & $900^{*}$ & 0.0017 & 942 & 943.3 & 83.904 & 908 & 908 \\
\hline frb $45-21-3 . m i s$ & 945,58245 & $900^{*}$ & 0.0015 & 941 & 943 & 479.1447 & 908 & 908 \\
\hline frb $45-21-4 . m i s$ & 945,58549 & $900^{*}$ & 0.0019 & 941 & 942.6 & 135.2191 & 907 & 907 \\
\hline frb $45-21-5 . m i s$ & 945,58579 & $900^{*}$ & 0.0019 & 943 & 943.7 & 235.1495 & 909 & 909 \\
\hline frb50-23-1.mis & 1150,80072 & $1100^{*}$ & 0.0016 & 1146 & 1147.6 & 409.0972 & 1109 & 1109 \\
\hline frb50-23-2.mis & 1150,80851 & $1100^{*}$ & 0.0018 & 1147 & 1148.6 & 87.4731 & 1110 & 1110 \\
\hline frb50-23-3.mis & 1150,81068 & $1100^{*}$ & 0.0021 & 1147 & 1148.1 & 414.1894 & 1110 & 1110 \\
\hline frb50-23-4.mis & 1150,80258 & $1100^{*}$ & 0.002 & 1147 & 1148.5 & 431.3235 & 1110 & 1110 \\
\hline frb50-23-5.mis & 1150,80035 & $1100^{*}$ & 0.002 & 1147 & 1148.3 & 837.1014 & 1109 & 1109 \\
\hline frb53-24-1.mis & 1272,94227 & $1219^{*}$ & 0.002 & 1269 & 1270.7 & 695.5386 & 1230 & 1230 \\
\hline frb53-24-2.mis & 1272,94289 & $1219^{*}$ & 0.0022 & 1269 & 1270.4 & 390.4401 & 1230 & 1230 \\
\hline frb53-24-3.mis & 1272,94127 & $1219^{*}$ & 0.002 & 1270 & 1271.2 & 552.5761 & 1229 & 1229 \\
\hline frb53-24-4.mis & 1272,94308 & $1219^{*}$ & 0.0024 & 1270 & 1270.4 & 588.6573 & 1229 & 1229 \\
\hline frb53-24-5.mis & 1272,94226 & $1219^{*}$ & 0.0022 & 1270 & 1270.6 & 95.6667 & 1230 & 1230 \\
\hline frb56-25-1.mis & 1400,109676 & $1344^{*}$ & 0.0022 & 1398 & 1399.1 & 90.5451 & 1357 & 1357 \\
\hline frb56-25-2.mis & 1400,109401 & $1344^{*}$ & 0.0022 & 1397 & 1397.3 & 412.38 & 1353 & 1353 \\
\hline frb56-25-3.mis & 1400,109379 & $1344^{*}$ & 0.0023 & 1397 & 1398.6 & 190.6417 & 1356 & 1356 \\
\hline frb56-25-4.mis & 1400,110038 & $1344^{*}$ & 0.0025 & 1398 & 1399 & 47.1687 & 1356 & 1356 \\
\hline frb56-25-5.mis & 1400,109601 & $1344^{*}$ & 0.0023 & 1397 & 1398.4 & 616.9286 & 1355 & 1355 \\
\hline frb59-26-1.mis & 1534,126555 & $1475^{*}$ & 0.0027 & 1533 & 1533.2 & 656.1494 & 1487 & 1487 \\
\hline frb59-26-2.mis & 1534,126163 & $1475^{*}$ & 0.0024 & 1531 & 1532.5 & 100.0605 & 1488 & 1488 \\
\hline frb59-26-3.mis & 1534,126082 & $1475^{*}$ & 0.0026 & 1531 & 1532.5 & 495.5281 & 1489 & 1489 \\
\hline frb59-26-4.mis & 1534,127011 & $1475^{*}$ & 0.0027 & 1531 & 1532.4 & 319.4073 & 1487 & 1487 \\
\hline frb59-26-5.mis & 1534,125982 & $1475^{*}$ & 0.0026 & 1533 & 1533.3 & 662.7202 & 1487 & 1487 \\
\hline
\end{tabular}


In Table 1, we present the experimental results on DIMACS benchmark. As is shown in the table, compared to the 2-Aprox algorithm, GRASP-CVC finds the better quality CVC solutions on all the 37 DIMACS instances. On most instances, the solutions found by GRASP-CVC are very close to the optimal $M V C$ and it even finds the same size solutions as optimal $M V C$ on 10 instances in a very short time, which means it finds the optimal CVC solutions on these 10 instances. Besides, the GRASP-CVC consumes very little time on most of the instances, which indicates the efficiency of the GRASP-CVC. Moreover, the GRASP-CVC takes a relatively long time on several larger instances (such as C2000.5.mis,DSJC1000.5.mis, C4000.5.mis and so on), this shows that the algorithm still has room for improvement. Another point worth noting is that though the 2-Aprox takes the less time on all of the 37 instances, most of its solutions are not so satisfactory compared to GRASP-CVC.

Table 2 provides the results on BHOSLIB benchmark. We can get the same conclusion as DIMACS that the GRASP-CVC performs best, even though it takes much more time than 2-Aprox. The solutions found by GRASP-CVC are close to the optimal $M V C$, which means the GRASP-CVC is very effective. The columns best and avg of GRASP-CVC have the same values on almost all of the instances, which means that the GRASP-CVC gets almost the same size solutions in ten times runs and this demonstrates the stability of the GRASP-CVC.

The comparison and experimental analyses above show that the GRASP-CVC has very good effectivity and efficiency for $C V C$ problem. It performs better than the competitive algorithm in solution quality. Moreover, the GRASP-CVC gets almost the same size solutions in ten times runs, which demonstrates the stability of the GRASP-CVC.

\section{Conclusion}

In this paper, a heuristic algorithm GRASP-CVC for connected vertex cover problem was proposed. A greedy function and a restricted candidate list $(R C L)$ were proposed to help to construct a high quality initial solution. Furthermore, the configuration checking $(C C)$ strategy was employed to reduce the cycling problem and improve the efficiency of the search. Experimental results demonstrate that GRASP-CVC works better than the comparison algorithm, which validates the effectiveness and efficiency of our GRASP-CVC solver. In the future, we will further study various heuristic methods and hope to design a more powerful heuristic algorithm to deal with CVC.

Acknowledgments: This work was fully supported by the National Natural Science Foundation of China under Grant No.61370156, No. 61403076, and No. 61403077; Research Fund for the Doctoral Program of Higher Education No. 20120043120017; Program for New Century Excellent Talents in University No. NCET-13-0724; the Large-scale Scientific Instrument and Equipment Sharing Project of Jilin Province (20150623024TC-03);The Natural Science Foundation for Youths of JiLin Province (20160520104JH).

\section{References}

1. Garey, M. R., Johnson, D. S. The rectilinear Steiner tree problem is NP-complete. SIAM Journal on Applied Mathematics,1997, 32(4), 826-834.

2. Moser, H. Exact algorithms for generalizations of vertex cover. Master's thesis, Fakultät für Mathematik und Informatik, Friedrich-Schiller-Universität Jena,2005.

3. Zhang, Z., Gao, X., Wu, W. PTAS for connected vertex cover in unit disk graphs. Theoretical Computer Science, 2009, 410(52), 5398-5402.

4. Guo, P., Wang, J., Geng, X. H., Kim, C. S., Kim, J. U. A variable threshold-value authentication architecture for wireless mesh networks. Journal of Internet Technology, 2014, 15(6), 929-935.

5. Shen, J., Tan, H. W., Wang, J., Wang, J. W., Lee, S. Y. A novel routing protocol providing good transmission reliability in underwater sensor networks. Journal of Internet Technology, 2015, 16(1), 171-178.

6. Priyadarsini, P. L. K., Hemalatha, T. Connected vertex cover in 2-connected planar graph with maximum degree 4 is NP-complete. International Journal of Mathematical, Physical and Engineering Sciences, 2008, 2(1), 51-54.

7. Fernau, H., Manlove, D. F. Vertex and edge covers with clustering properties: Complexity and algorithms. Journal of Discrete Algorithms, 2009, 7(2), 149-167. 
8. Watanabe, T., Kajita, S., Onaga, K. Vertex covers and connected vertex covers in 3-connected graphs. IEEE International Sympoisum on Circuits and Systems. IEEE, 1991, vol.2, 1017-1020.

9. Mölle, D., Richter, S., Rossmanith, P. Enumerate and expand: Improved algorithms for connected vertex cover and tree cover. Theory of Computing Systems, 2008, 43(2), 234-253.

10. Binkele-Raible, D. Amortized analysis of exponential time-and parameterized algorithms: Measure \& Conquer and Reference Search Trees. Praca doktorska, University of Trier, Trier, Germany,2010.

11. Savage, C. Depth-first search and the vertex cover problem. Information Processing Letters, 1982, 14(5), 233-235.

12. Fujito, T., Doi, T. A 2-approximation NC algorithm for connected vertex cover and tree cover. Information processing letters, 2004, 90(2), 59-63.

13. Escoffier, B., Gourvès, L., Monnot, J. Complexity and approximation results for the connected vertex cover problem in graphs and hypergraphs. Journal of Discrete Algorithms, 2010, 8(1), 36-49.

14. Cardinal, J., Levy, E. Connected vertex covers in dense graphs. Lecture Notes in Computer Science, 2008, 5171, 35-48.

15. Li, Y., Yang, Z., Wang, W. Complexity and algorithms for the connected vertex cover problem in 4-regular graphs. Applied Mathematics and Computation,2017, 301, 107-114.

16. Resende, M. G., Ribeiro, C. C. GRASP: Greedy randomized adaptive search procedures. In Search methodologies, 2014, pp. 287-312, Springer US.

17. Zhou, Y., Zhang, H., Li, R., Wang, J. Two local search algorithms for partition vertex cover problem. Journal of Computational and Theoretical Nanoscience, 2016, 13(1), 743-751.

18. Li, X., Zhang, J., Yin, M. Animal migration optimization: an optimization algorithm inspired by animal migration behavior. Neural Computing and Applications, 2014, 24(7-8), 1867-1877.

19. Wang, Y., Cai, S., Yin, M. Two Efficient Local Search Algorithms for Maximum Weight Clique Problem. In AAAI,2016, pp.805-811.

20. Wang, Y.,Yin, M., Ouyang, D., Zhang, L. A novel local search algorithm with configuration checking and scoring mechanism for the set k-covering problem. International Transactions in Operation Research, 2017, 24(26),1436-1485.

21. Cai, S., Su, K., Sattar, A. Local search with edge weighting and configuration checking heuristics for minimum vertex cover. Artificial Intelligence, 2011, 175(9-10), 1672-1696.

22. Luo, C., Cai, S., Wu, W., Su, K. Double Configuration Checking in Stochastic Local Search for Satisfiability. In AAAI, 2014,pp.2703-2709.

23. Luo, C., Cai, S., Wu, W., Jie, Z., Su, K. CCLS: an efficient local search algorithm for weighted maximum satisfiability. IEEE Transactions on Computers, 2015, 64(7), 1830-1843.

24. Gao, J., Wang, J., Yin, M. Experimental analyses on phase transitions in compiling satisfiability problems. Science China Information Sciences, 2015, 58(3), 1-11.

25. Cai, S., Su, K., Luo, C., Sattar, A. NuMVC: An efficient local search algorithm for minimum vertex cover. Journal of Artificial Intelligence Research, 2013, 46, 687-716. 